\title{
Síntese e avaliação do prop-2-in-1-il 4,6-di-O-acetil-2,3- didesoxi- $\alpha-D$-eritro-hex-2-enopiranosídeo contra espécies não albicans de Candida e resultados da associação com a anfotericina B ou com o cetoconazol
}

\section{Synthesis and evaluation of prop-2-yn-1-yl 4,6-di-O-acetyl-2,3-dideoxy- $\alpha$-D-erythro- hex-2-enopyranoside against non-albicans species of Candida, and effectiveness of amphotericin B or ketoconazole combination}

\author{
Gustavo Lima Soares , Cosme Silva Santos², Juliano Carlo Rufino de Freitas ${ }^{1,2}$, Wylly Araújo de Oliveira \\ ' Universidade Federal de Campina Grande, Centro de Educação e Saúde, Unidade Acadêmica de Saúde, Cuité, Paraíba, Brasil \\ ${ }_{2}^{2}$ Universidade Federal Rural de Pernambuco, Departamento de Química, Recife, Pernambuco, Brasil
}

\begin{abstract}
RESUMO
OBJETIVOS: Sintetizar e avaliar a ação antifúngica do prop-2-in-1-il 4,6-di-O-acetil-2,3-didesoxi- $\alpha$-D-eritro-hex-2enopiranosídeo contra as espécies não albicans de Candida, assim como avaliar sua atividade antimicrobiana quando associado com a anfotericina B ou com o cetoconazol. MATERIAIS E MÉTODOS: A síntese desse glicosídeo foi obtida por meio da reação de glicosilação empregando-se o tri-O-acetil-D-glucal, o álcool 2-propin-1-ol e a montmorillonita K-10. O teste da atividade antifúngica foi realizado pelo método da microdiluição em caldo, e a associação com a anfotericina B ou com o cetoconazol, pelo método de Checkerboard. RESULTADOS: O glicosídeo foi obtido com $95 \%$ de rendimento após o tempo reacional de $1 \mathrm{~h}$ e não apresentou atividade antifúngica nas concentrações testadas $(512 ; 0,5 \mu \mathrm{g} / \mathrm{mL})$ quando ensaiado isoladamente. Quando associado com a anfotericina B (concentração inibitória mínima - CIM $2 \mu \mathrm{g} / \mathrm{mL}$ ) ou com o cetoconazol (CIM 0,5 $\mu \mathrm{g} / \mathrm{mL}$ ), observou-se sinergismo e indiferença, respectivamente. CONCLUSÃO: Verificou-se que o glicosídeo 3 obtido apresentou excelente rendimento na síntese, mas não demonstrou atividade antifúngica ante as cepas não albicans. Quando associado com a anfotericina B, a atividade foi promissora; com o cetaconazol, indiferente.
\end{abstract}

Palavras-chave: Candida; Glicosídeo; Combinação de Medicamentos; Antifúngicos.

\begin{abstract}
OBJECTIVES: To synthesize and evaluate the antifungal action of prop-2-yn-1-yl 4,6-di-O-acetyl-2,3dideoxy- $\alpha$-D-erythro-hex-2-enopyranoside against non-albicans species of Candida as well as to evaluate its antimicrobial activity when associated with amphotericin B or with ketoconazole. MATERIALS AND METHODS: The synthesis of this glycoside was obtained by the glycosylation reaction using tri-O-acetyl-D-glucal, 2-propyn-1-ol alcohol and montmorillonite K-10. The antifungal activity test was performed by the broth microdilution method and the association with amphotericin B or ketoconazole by the checkerboard method. RESULTS: The glycoside was obtained with $95 \%$ yield after the reaction time of $1 \mathrm{~h}$ and did not present antifungal activity at the tested concentrations $(512$; $0.5 \mu \mathrm{g} / \mathrm{mL}$ ) when tested alone. When combined with amphotericin B (minimum inhibitory concentration - MIC 2 $\mu \mathrm{g} / \mathrm{mL}$ ) or ketoconazole (MIC $0.5 \mu \mathrm{g} / \mathrm{mL}$ ), synergism and indifference were observed respectively. CONCLUSION: Glycoside 3 was obtained in excellent yield and did not present antifungal activity against the strains of non-albicans species. But, when associated with amphotericin B, the activity was promising; with ketoconazole, indifferent.
\end{abstract}

Keywords: Candida; Glycoside; Combination of Drugs; Antifungals.

\footnotetext{
Correspondência / Correspondence:

Wylly Araújo de Oliveira

Universidade Federal de Campina Grande, Centro de Educação e Saúde

Sítio Olho D'água da Bica, s/n. CEP: 58175-000 - Cuité, Paraíba, Brasil - Tel.: +55 (83) 3372-1973

E-mail:wyllyoliveira@gmail.com
} 


\section{INTRODUÇÃO}

gênero Candida apresenta elevadas taxas de colonização e infecção'. Esses micro-organismos são leveduras cosmopolitas encontradas na microbiota normal do homem e de animais, estando presente em diversos sítios do corpo, como mucosas do trato gastrintestinal, boca, vagina e pele ${ }^{2}$; entretanto, tornam-se patogênicas em casos de alterações nos mecanismos de defesa do indivíduo?.

As infecções ocasionadas por Candida englobam casos de doenças superficiais e invasivas, sobretudo quando ocorre o acometimento de pacientes expostos a diversos fatores de riscos. Infecções de pele, mucosas, candidíase vaginal e candidemias são regularmente relatadas'. Em pacientes soropositivos, a candidíase orofaríngea é a infecção fúngica mais habitual ${ }^{3}$.

Durante a evolução da infecção por Candida, a levedura atua expressando diversos fatores de virulência, dentre os quais destacam-se a produção de enzimas extracelulares, a síntese de hemolisinas, a capacidade de adesão e a formação de biofilme ${ }^{4}$. Tais fatores são definidos geneticamente, entretanto são expressos pelos micro-organismos quando expostos a determinadas condições ${ }^{5}$. $\bigcirc$ interesse nesses fatores de virulência justifica-se pela necessidade de se estabelecerem possíveis tratamentos e formas de prevenir a infecção ${ }^{6}$.

A constante utilização de agentes antifúngicos tem despertado preocupações devido à seleção de cepas resistentes. Estudos têm relatado alta incidência de infecções ocasionadas por leveduras do gênero Candida resistentes aos fármacos de primeira e segunda escolha, como é o caso dos derivados azólicos, que estão entre os mais prescritos para 0 tratamento de candidíase ${ }^{7,8}$.

tratamento de patógenos recorrentes é uma problemática em ascensão nos dias atuais e fundamenta-se em duas alternativas: a descoberta de novos fármacos antimicrobianos e/ou a associação de substâncias já existentes ${ }^{9}$. A associação de antimicrobianos é uma alternativa em infecções de etiologia polimicrobiana, onde a obtenção da cura pela monoterapia é dificultada ${ }^{10}$.

A investigação de novas moléculas, com potencial de serem ativas biologicamente, desponta como uma importante alternativa terapêutica, possibilitando explorar novos alvos terapêuticos e propor novas estratégias de agir sobre alvos terapêuticos já determinados ${ }^{11}$. Uma grande quantidade de moléculas ativas vem sendo obtida pela síntese orgânica ${ }^{12}$, como é o caso dos glicosídeos ${ }^{13,14,15}$.

Os glicosídeos são produtos obtidos por meio da reação de glicosilação, constituídos basicamente por duas unidades, a unidade sacarídica e a unidade ligada pelo carbono anomérico, denominada glicona (grupo sacarídico) ou aglicona (grupo não sacarídico).

Os objetivos deste estudo foram sintetizar e avaliar a ação antifúngica do prop-2-in-1-il 4,6-di-Oacetil-2,3-didesoxi- $\alpha$-D-eritro-hex-2-enopiranosídeo contra espécies não albicans de Candida, assim como avaliar sua atividade antimicrobiana quando associado à anfotericina $\mathrm{B}$ ou ao cetoconazol.

\section{MATERIAIS E MÉTODOS}

\section{PROCEDIMENTO GERAL: EQUIPAMENTOS E ANÁLISE DOS DADOS}

As análises de ressonância magnética nuclear (RMN) foram realizadas em um espectrômetro VARIAN ${ }^{\circledR}$ modelo Unity Plus-300, utilizando como solvente o clorofórmio deuterado $\left(\mathrm{CDCl}_{3}\right)$. Esse espectrômetro foi calibrado usando tetrametilsilano $(0,00 \mathrm{ppm})$ como referência interna para os núcleos de ${ }^{1} \mathrm{H}$ e ${ }^{13} \mathrm{C}$, e todas as constantes de acoplamento $(J)$ foram descritas em hertz $(\mathrm{Hz})$. $\mathrm{O}$ espectro de infravermelho foi registrado em um espectrofotômetro de infravermelho com transformada de Fourier no Spectrum 400 FT-IR/FT-NIR Spectrometer, modelo PerkinElmer, sendo a amostra preparada como pastilhas de $\mathrm{KBr}$. A rotação específica foi determinada em um polarímetro digital da marca JASCO ${ }^{\circledR}$ P-2000 equipado com a luz de sódio em comprimento de onda $589 \mathrm{~nm}$. A amostra foi preparada em concentração de $1 \mathrm{~m} / \mathrm{v} \%$ (em $\mathrm{MeOH}$ ) em uma cubeta de $1 \mathrm{~mL}$. $\bigcirc$ ponto de fusão foi realizado no Electrothermal série IA9100 Digital Melting Point. A chapa de agitação com aquecimento e a manta aquecedora eram da marca Fisatom, modelo 754A e 102E, respectivamente. $O$ solvente foi removido, utilizando um evaporador rotativo da BÜCHI Rotavapor ${ }^{\circledR}$, modelo R-114, conectado a uma bomba de vácuo modelo KNF Neuberger, e o solvente remanescente foi removido, utilizando uma bomba de alto vácuo da Edwards, modelo RV3. As estruturas dos compostos foram geradas por meio do software ChemBioUltra $^{\circledR}$ v12.0.

PROCEDIMENTO DE SÍNTESE DO prop-2-in-1-il 4,6-diO-acetil-2,3-didesoxi- $\alpha$-D-eritro-hex-2-enopiranosídeo (GLICOSÍDEO 3)

monitoramento das reações foi realizado por cromatografia em camada delgada, utilizando placas de sílica-gel contendo indicador fluorescente $F_{254}$ da Merck. Para visualização, as placas foram colocadas em solução ácida $\left(E \nmid O H / H_{2} \mathrm{SO}_{4}\right.$, 95:5) e solução de vanilina. A purificação, por meio da cromatografia líquida em coluna, foi realizada com o uso de sílica-gel 60 (Merck, 70-230 mesh), como fase estacionária, e sistemas hexano:acetato de etila, como fase móvel, numa coluna de vidro em diferentes proporções. Os solventes comerciais foram purificados de acordo com os protocolos descritos na literatura ${ }^{16}$. $O$ hexano e o acetato de etila foram destilados em coluna de Vigreaux, o diclorometano, sob hidreto de cálcio, e o metanol, sob magnésio metálico e iodo sublimado. Os reagentes tri-O-acetil-D-glucal e o álcool 2-propin-1-ol da Sigma-Aldrich.

A síntese do composto prop-2-in-1-il 4,6-di-O-acet il-2,3-didesoxi- $\alpha$-D-eritro-hex-2-enopiranosídeo foi realizada segundo o protocolo de Toshima et al. ${ }^{17}$, com algumas modificações. Desse modo, em um balão de fundo redondo, com capacidade de $50 \mathrm{~mL}$, 
foram adicionados o tri-O-acetil-D-glucal $(1 ; 0,27$ g; $1 \mathrm{mmol})$, o álcool 2-propin-1-ol $(2 ; 69,7 \mu \mathrm{L} ; 67,2$ $\mathrm{mg} ; 1,2 \mathrm{mmol})$ e o diclorometano seco $(20 \mathrm{~mL})$. Em seguida, o balão foi resfriado a $0{ }^{\circ} \mathrm{C}$ e mantido sob agitação por $5 \mathrm{~min}$. Após esse intervalo de tempo, foi adicionado montmorillonita $\mathrm{K}-10(0,16 \mathrm{~g} ; 60 \% \mathrm{M} / \mathrm{M})$, e um sistema de refluxo foi acoplado ao balão. A mistura reacional foi mantida sob agitação e aquecimento $\left(50 \pm 5^{\circ} \mathrm{C}\right)$ por 1 h. Logo depois, o aquecimento foi interrompido, e o término da reação, verificado por cromatografia de camada delgada. Dando prosseguimento, a solução contida no balão foi filtrada e o solvente, removido sob pressão reduzida, utilizando evaporador rotativo. $O$ produto bruto foi purificado em coluna cromatográfica, utilizando sílica-gel como fase estacionária e o sistema hexano:acetato de etila (início de 100:0 até 90:10) como fase móvel.

\section{DETERMINAÇÃO DA CONCENTRAÇÃO INIBITÓRIA MÍNIMA}

Foram utilizadas quatro cepas fúngicas: Candida tropicalis ATCC-13803, Candida parapsilosis ATCC-20019, Candida krusei LM-13 e Candida guilliermondii LM-703.

A determinação da concentração inibitória mínima (CIM) foi realizada pela técnica da microdiluição em caldo, utilizando como meio o caldo Sabouraud dextrose ${ }^{2,18,19}$. A concentração inicial do prop-2-in-1-il 4,6-di-O-acetil-2,3-didesoxi- $\alpha$-D-eritro-hex- 2 enopiranosídeo testada foi de $512 \mu \mathrm{g} / \mathrm{mL}$ e diluída seriadamente 1:2 até a concentração final de $0,5 \mu \mathrm{g} / \mathrm{mL}$. As concentrações iniciais da anfotericina $\mathrm{B}$ e do cetoconazol foram de $128 \mu \mathrm{g} / \mathrm{mL}$ e diluídas seriadamente 1:2 até a concentração final de $0,0078 \mu \mathrm{g} / \mathrm{mL}$. O inóculo foi adicionado em uma concentração final de aproximadamente 1-5 $\mathrm{x}$ $10^{5} \mathrm{UFC} / \mathrm{mL}$ em cada cavidade. As soluções foram preparadas com o auxílio de DMSO (dimetilsulfóxido). Foram utilizados controles com DMSO nas mesmas concentrações usadas no teste.

Utilizou-se inóculo fúngico, contendo aproximadamente 1-5 × $10^{6} \mathrm{UFC} / \mathrm{mL}$, padronizado de acordo com a turbidez do tubo 0,5 da escala de McFarland. Todos os testes foram realizados em triplicata.

A incubação dos ensaios ocorreu em estufa a aproximadamente $35{ }^{\circ} \mathrm{C}$, durante $48 \mathrm{~h}$. Como
CIM, considerou-se a menor concentração da substância testada, capaz de produzir inibição visual do crescimento das cepas de levedura utilizadas nos ensaios microbiólogos ${ }^{20}$; fato confirmado pela adição de $20 \mu \mathrm{L}$ de TTC 1\% (2,3,5 trifenil cloreto de tetrazólio) em cada cavidade? .

\section{ASSOCIAÇÃO DAS DROGAS}

Para a associação das substâncias, foram preparadas, separadamente, diferentes soluções nas concentrações de $\mathrm{CIM} \times 8, \mathrm{CIM} 4$, CIM×2, CIM, CIM/2, $\mathrm{CIM} / 4$ e $\mathrm{CIM} / 8$.

Realizou-se o estudo das associações pela técnica Checkerboard para a determinação do índice da concentração inibitória fracionária (ICIF), utilizando placas estéreis de microdiluição com 96 cavidades. Distribuíram-se as soluções na placa, de forma que todas as concentrações da substância A (glicosídeo), na horizontal, combinassem com todas as concentrações da substância B (anfotericina B ou cetoconazol) na vertical.

O experimento foi conduzido com inóculo 1-5 x $10^{5} \mathrm{UFC} / \mathrm{mL}$ em cada cavidade. Para a realização das associações, utilizou-se a cepa fúngica $C$. tropicalis ATCC-13803.

Os ensaios foram incubados em estufa a $35^{\circ} \mathrm{C}$ por 48 h, e, após esse período, determinada a CIM da associação.

Calculou-se o ICIF pela seguinte equação: ICIF $=$ $\mathrm{CIF}^{A}+\mathrm{ClF}^{B}$, onde a concentração inibitória fracionária ${ }^{A}$ $\left(\mathrm{ClF}^{\mathrm{A}}\right)$ foi calculada pela relação $\mathrm{CIM}^{A}$ combinado/ $\mathrm{CIM}^{\mathrm{A}}$ sozinho; enquanto que o $\mathrm{CIF}^{B}=\mathrm{CIM}^{\mathrm{B}}$ combinado $/ \mathrm{CIM}^{\mathrm{B}}$ sozinho ${ }^{21}$. Sendo A o glicosídeo e B a anfotericina B ou o cetoconazol.

O ICIF foi classificado da seguinte forma: sinergismo $(\leq 0,5)$, indiferença $(>0,5<4)$, antagonismo $(\geq 4)^{22,23}$

\section{RESULTADOS}

O prop-2-in-1-il 4,6-di-O-acetil-2,3-didesoxi- $\alpha$-Deritro-hex-2-enopiranosídeo (glicosídeo 3) foi obtido na forma de um sólido branco amorfo, com excelente rendimento (Figura 1).

Os resultados da análise espectroscópica dos espectros de $\mathrm{RMN}{ }^{1} \mathrm{H}, \mathrm{RMN}{ }^{13} \mathrm{C}$ e infravermelho estão descritos na tabela 1.

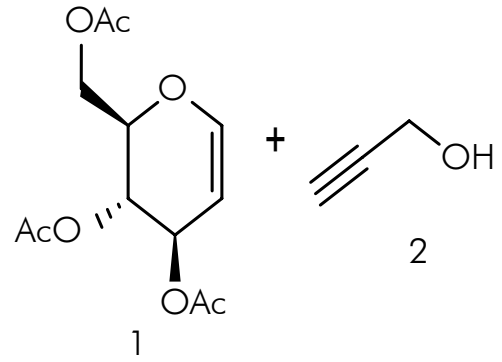

1

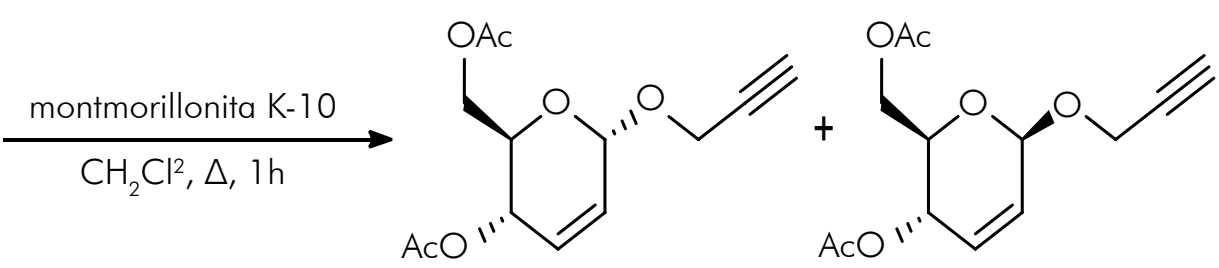

3

Figura 1 - Síntese dos $O$-glicosídeos 2,3-insaturados 3 e 4 
$\mathrm{O}$ espectro de $\mathrm{RMN}{ }^{13} \mathrm{C}$ do glicosídeo 3 apresentou 13 sinais quimicamente diferentes (Tabela 1), valor esse semelhante à quantidade de carbonos presentes na estrutura do glicosídeo 3.

Na figura 2, observa-se a ampliação do espectro de RMN ${ }^{1} \mathrm{H}\left(\mathrm{CDCl}_{3}, 300 \mathrm{MHz}\right)$ da reação de glicosilação para determinar a proporção dos glicosídeos 3 e 4 .
A técnica espectroscópica NOESY corroborou a constatação do produto majoritário da reação de glicosilação (Figura 3).

glicosídeo 3 usado nos ensaios não apresentou atividade antifúngica em qualquer das cepas utilizadas nas concentrações testadas. Os valores das CIM da anfotericina $B$ e do cetoconazol estão descritas na tabela 2.

Tabela 1 - Análise espectroscópica dos espectros de RMN ${ }^{1} \mathrm{H}, \mathrm{RMN}{ }^{13} \mathrm{C}$ e infravermelho do glicosídeo 3

\begin{tabular}{|c|c|c|c|}
\hline $\begin{array}{c}\text { Estrutura do } \\
\text { composto }\end{array}$ & $\begin{array}{c}\mathrm{RMN}^{\mathrm{H}} \mathrm{H} \\
\left(\mathrm{CDCl}_{3^{\prime}}, 300 \mathrm{MHz}\right) \\
\mathrm{ppm}\end{array}$ & $\begin{array}{c}\mathrm{RMN}^{13} \mathrm{C} \\
\left(\mathrm{CDCl}_{3^{\prime}} 75 \mathrm{MHz}\right) \\
\mathrm{ppm}\end{array}$ & $\begin{array}{c}\text { Infravermelho } \\
\text { (pastilha KBr) } \\
\mathrm{cm}^{-1}\end{array}$ \\
\hline $\mathrm{AcO}_{3}$ & $\begin{array}{l}5,90(d l, J=10,2 \mathrm{~Hz}, \mathrm{H}-3) \\
5,82(d t, J=10,2 \text { e } 1,5 \mathrm{~Hz}, \mathrm{H}-2) \\
5,33(d d d, J=9,6,3,0 \text { e } 1,5 \mathrm{~Hz}, \mathrm{H}-4) \\
5,22(\mathrm{sl}, \mathrm{H}-1) \\
4,29(d, J=2,4 \mathrm{~Hz}, \mathrm{H}-7) \\
4,25(d d, J=12,4 \text { e } 5,4 \mathrm{~Hz}, \mathrm{H}-6) \\
4,16(d d, J=12,4 \text { e } 2,4 \mathrm{~Hz}, \mathrm{H}-6) \\
4,07(d d d, J=9,6 ; 5,4 \text { e } 2,4 \mathrm{~Hz}, \mathrm{H}-5) \\
2,07(t, J=2,4 \mathrm{~Hz}, \mathrm{H}-9) \\
2,09(s, O A c) \\
2,07(s, O A c)\end{array}$ & $\begin{array}{c}170,7(\mathrm{C}=\mathrm{O}) \\
170,2(\mathrm{C}=\mathrm{O}) \\
129,7(\mathrm{C}-3) \\
127,1(\mathrm{C}-2) \\
92,7(\mathrm{C}-1) \\
78,9(\mathrm{C}-8) \\
74,8(\mathrm{C}-9) \\
67,1(\mathrm{C}-5) \\
65,0(\mathrm{C}-4) \\
62,7(\mathrm{C}-6) \\
55,0(\mathrm{C}-7) \\
20,9\left(\mathrm{CH}_{3}\right) \\
20,7\left(\mathrm{CH}_{3}\right)\end{array}$ & $\begin{array}{l}3296(v \mathrm{Csp}-\mathrm{H}) \\
3058\left(v \mathrm{Csp}^{2}-\mathrm{H}\right) \\
2918\left(\mathrm{csp}^{3}-\mathrm{H}\right) \\
2129(v \mathrm{C} \equiv \mathrm{C}) \\
1741(\mathrm{vC}=\mathrm{O}) \\
1373\left(\delta \mathrm{CH}_{3}\right) \\
1235(\delta \mathrm{O}-\mathrm{C}-\mathrm{C}) \\
1038(\delta \mathrm{C}-\mathrm{O})\end{array}$ \\
\hline
\end{tabular}

RMN: Ressonância magnética nuclear; ppm: Parte por milhão; dl: Dupleto largo; dt: Duplo tripleto; ddd: Duplo dupleto de dupleto; sl: Simpleto largo; d: Dupleto; t: Tripleto; s: Simpleto; v: Deformação axial; $\delta$ : Deformação angular.

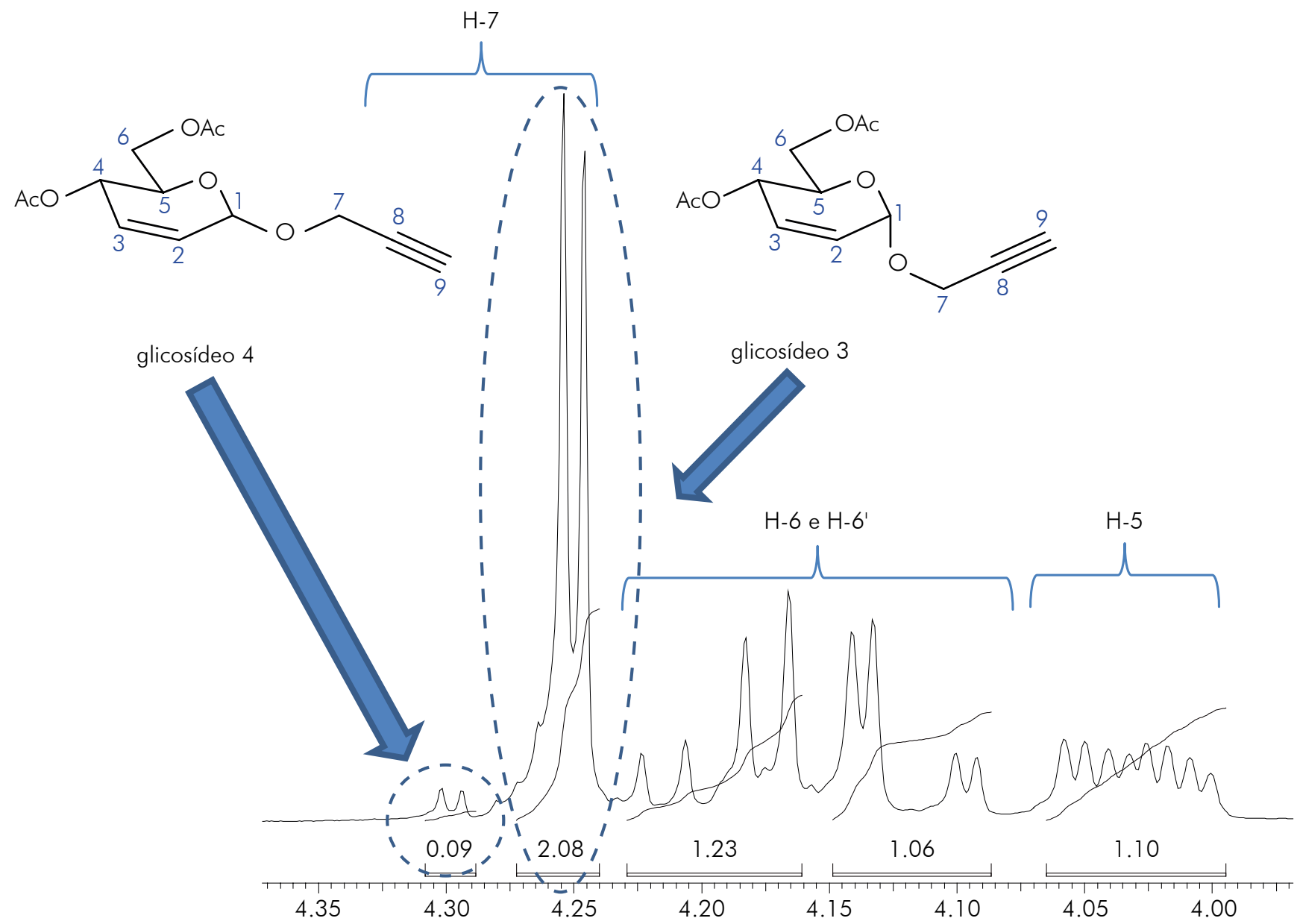

Figura 2 - Ampliação do espectro de $\mathrm{RMN}{ }^{1} \mathrm{H}\left(\mathrm{CDCl}_{3}, 300 \mathrm{MHz}\right)$ da reação de glicosilação para determinar a proporção dos glicosídeos 3 e 4 


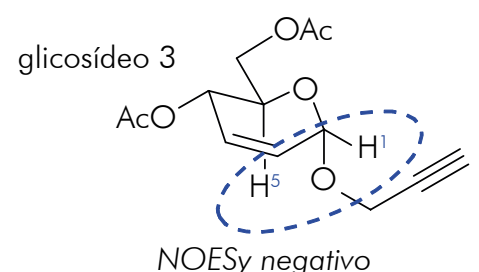

NOESy negativo

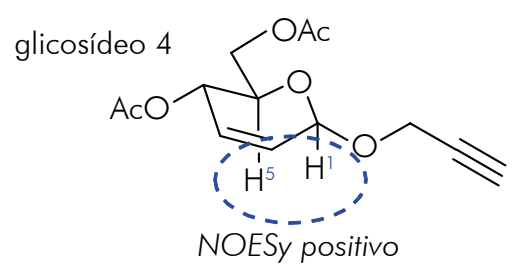

$\mathrm{H}-7$

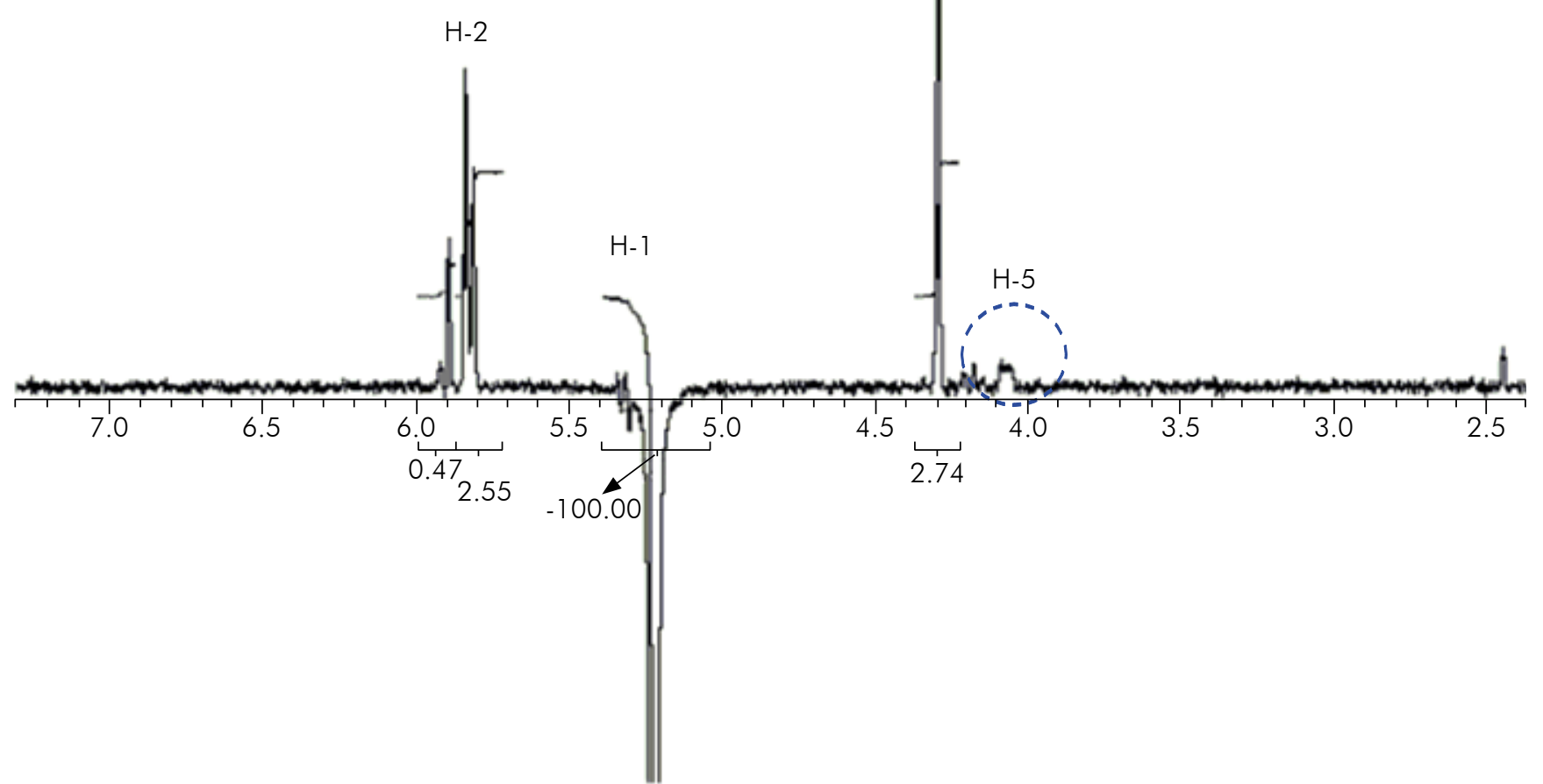

Figura 3 - Espectro de NOESY-1D (300 MHz, CDCl3) do glicosídeo 3 - irradiação no hidrogênio H-1 (5,22 ppm)

Tabela 2 - Valores das CIM do glicosídeo 3, da anfotericina B e do cetoconazol frente às cepas não albicans

\begin{tabular}{lccc}
\hline \multicolumn{1}{c}{ Espécie fúngica } & $\begin{array}{c}\text { CIM } \\
\text { glicosídeo 3 }\end{array}$ & $\begin{array}{c}\text { CIM } \\
\text { anfotericina B }\end{array}$ & $\begin{array}{c}\text { CIM } \\
\text { cetoconazol }\end{array}$ \\
\hline C. krusei LM-13 & + & $2 \mu \mathrm{g} / \mathrm{mL}$ & $0,5 \mu \mathrm{g} / \mathrm{mL}$ \\
C. parapsilosis ATCC-20019 & + & $2 \mu \mathrm{g} / \mathrm{mL}$ & $0,5 \mu \mathrm{g} / \mathrm{mL}$ \\
C. tropicalis ATCC-13803 & + & $2 \mu \mathrm{g} / \mathrm{mL}$ & $0,5 \mu \mathrm{g} / \mathrm{mL}$ \\
C. guilliermondii LM-703 & + & $0,5 \mu \mathrm{g} / \mathrm{mL}$ & $0,125 \mu \mathrm{g} / \mathrm{mL}$ \\
\hline
\end{tabular}

CIM: Concentração inibitória mínima; +: Crescimento fúngico em todas as concentrações testadas.

Para a realização das associações, foi considerada, como CIM do glicosídeo 3, a maior concentração testada. A associação entre o glicosídeo 3 e a anfotericina $B$ apresentou resultado muito satisfatório e promissor; enquanto que, entre o glicosídeo 3 e o cetoconazol, o resultado não foi o esperado (Tabela 3).

Tabela 3 - Associação entre o glicosídeo 3 e a anfotericina B ou o cetoconazol contra C. tropicalis ATCC-13803

$\begin{array}{clcl}\text { CIF anfotericina B } & 0,125 & \text { CIF cetoconazol } & 0,5 \\ \text { CIF glicosídeo 3 } & 0,125 & \text { CIF glicosídeo 3 } & 0,125 \\ \text { ICIF } & 0,25 & \text { ICIF } & 0,625\end{array}$




\section{DISCUSSÃO}

A análise dos dados espectroscópicos, provenientes dos espectros de RMN 'H e de NOESY, demonstrou que o glicosídeo, obtido como produto majoritário da reação de glicosilação, foi o composto 3. As informações detalhadas na tabela 1 corroboraram a estrutura do glicosídeo 3. Por fim, o ângulo de desvio óptico da solução metanólica do glicosídeo 3 foi positivo.

Após algumas mudanças no protocolo experimental descrito por Toshima et al. ${ }^{17}$, os glicosídeos 3 e 4 foram obtidos com $95 \%$ de rendimento e com uma diastereosseletividade de 95:5, que foi um pouco superior ao relatado por aqueles autores, tendo sido determinada pelo espectro de $\mathrm{RMN}{ }^{1} \mathrm{H}$, utilizando a razão entre as integrais do sinal do hidrogênio metilênico (numerado como $\mathrm{H}-7$ ) de ambos os compostos. $\mathrm{O}$ hidrogênio $\mathrm{H}-7$ foi escolhido porque os demais sinais estavam sobrepostos (por exemplo, $\mathrm{H}-6$, $\mathrm{H}-6$ ' e $\mathrm{H}-5$ ) (Figura 2).

Por meio da técnica espectroscópica NOESY, que leva em consideração as proximidades dos núcleos de ${ }^{1} \mathrm{H}$ no espaço ${ }^{24}$, foi observado que o produto majoritário foi o glicosídeo 3, uma vez que a irradiação seletiva no hidrogênio $\mathrm{H}-1$ (5,22 ppm) não afetou consideravelmente o hidrogênio $\mathrm{H}-5$ (4,07ppm), apenas os hidrogênios H-7 (4,29 ppm) e H-2 (5,82 ppm) (Figura 3).

Com relação ao espectro de infravermelho do glicosídeo 3, foram observadas várias bandas de absorção; no entanto, as bandas em número de ondas de 3296, 3058, 2918, 2129, 1741, 1373, 1235 e $1038 \mathrm{~cm}^{-1}$ foram atribuídas às ligações Csp-H (deformação axial, moderada), $\mathrm{Csp}^{2}-\mathrm{H}$ (deformação axial, fraca), $\mathrm{Csp}^{3}-\mathrm{H}$ (deformação axial assimétrica, fraca), $\mathrm{C} \equiv \mathrm{C}$ (deformação axial, fraca), $\mathrm{C}=\mathrm{O}$ (deformação axial, forte), $\mathrm{CH}_{3}$ (deformação angular, moderada), O-CO-C (deformação angular, moderada) e C-O (deformação angular, forte), respectivamente.

$\bigcirc$ ponto de fusão e o ângulo de desvio óptico da solução metanólica do glicosídeo 3 foram de 58 a $59{ }^{\circ} \mathrm{C}$ e $[\alpha]_{D}^{20}+137,7$, valores esses próximos ao descrito na literatura $\left(59^{\circ} \mathrm{C}\right)^{25}$.

A terapia combinada de antifúngicos é considerada uma alternativa terapêutica importante para o tratamento de infecções, possibilitando benefícios como: redução da toxicidade, devido ao uso de menores concentrações de cada substância ativa; tratamento das infecções por múltiplos patógenos; e/ou prevenção ou retardo do desenvolvimento in vivo de subpopulações resistentes a antimicrobianos ${ }^{26,27,28}$. Nesse contexto, na combinação de fármacos, o sinergismo é a interação mais desejada ${ }^{27,29}$.

Estudos relatam que $\mathrm{O}$ efeito antimicrobiano dos fármacos tem sido potencializado tanto em micro-organismos sensíveis quanto em cepas resistentes a cada um dos fármacos, nos casos de associações sinérgicas ${ }^{10,30}$
Para que se estabeleça a terapia combinada adequada entre antimicrobianos, é fundamental que se compreenda a interação existente na combinação dos fármacos. A metodologia do Checkerboard é uma das mais utilizadas para verificar o comportamento da associação in vitro no crescimento microbiano ${ }^{27}$.

Segundo Liu et al. ${ }^{31}$, existem limitações nas combinações de antifúngicos, como alto custo, efeitos indesejáveis e resultados contraditórios de ações sinérgicas ou antagônicas de algumas combinações de antifúngicos. Dessa forma, estudos com foco em avaliar combinações de antifúngicos com não antifúngicos vem sendo desenvolvidos.

A combinação de antifúngicos com agentes não antifúngicos, a exemplo da combinação com antibacterianos, sobretudo em infecções fúngicas profundas, apresenta-se como uma importante alternativa terapêutica. Outros agentes não antifúngicos, como os inibidores da calcineurina, inibidores da proteína 90 de choque térmico, reguladores da homeostase do cálcio e anti-inflamatórios não esteroidais, têm sido combinados com fluconazol, observando-se resultados sinérgicos. Outro fato importante é que combinações in vitro do ibuprofeno com o fluconazol contra estirpes resistentes ao fluconazol apresentam sinergia; porém essa mesma ação não é observada em estirpes sensíveis ao fluconazol ${ }^{31}$.

Foi observado sinergismo entre a associação de concentrações subinibitórias de estatinas com antifúngicos da classe dos azóis contra as espécies não albicans. A fluocitosina é um exemplo de antifúngico de pouca utilização clínica isoladamente, devido ao seu limitado espectro de ação e toxicidade considerável $^{32,33}$. Entretanto, é comumente utilizada em combinação com a anfotericina $B^{34}$. Estudos da associação entre a anfotericina $B$ e a fluocitosina têm demonstrado sinergia contra cepas resistentes de Candida spp. ${ }^{35}$.

Os resultados da associação do glicosídeo com a anfotericina B foram diferentes da associação com - cetoconazol, possivelmente devido às diferenças entre os mecanismos de ação dos poliênicos e dos azóis.

\section{CONCLUSÃO}

- glicosídeo 3 obtido apresentou excelente rendimento e sua estrutura foi confirmada por diferentes técnicas espectroscópicas; no entanto, não apresentou atividade antifúngica ante as cepas não albicans de Candida quando utilizado isoladamente. Quando associado com a anfotericina B, a atividade foi sinérgica; com o cetaconazol, indiferente.

\section{AGRADECIMENTOS}

Os autores agradecem a Universidade Federal de Campina Grande e a Central Analítica do Departamento de Química Fundamental da Universidade Federal de Pernambuco. 


\section{APOIO FINANCEIRO}

O presente trabalho foi realizado com o apoio do Conselho Nacional de Desenvolvimento Científico e Tecnológico.

\section{CONFLITOS DE INTERESSE}

Os autores declaram que não houve conflitos de interesse.

\section{CONTRIBUIÇÃO DOS AUTORES}

Todos os autores contribuíram com a idealização do estudo, a análise e a interpretação dos dados e com a redação do manuscrito, aprovando a versão final publicada. Declaram-se responsáveis pelo conteúdo integral do artigo, garantindo sua precisão e integridade.

\section{REFERÊNCIAS}

1 Dignani MC, Solomkin JS, Anaissie E. Candida. In: Anaissie E, McGinnis MR, Pfaller MA, editors. Medical mycology. Philadelphia: Churchill Livingstone; 2003. p. 195-239.

2 Castro RD, Lima EO. Atividade antifúngica dos óleos essenciais de sassafrás (Ocotea odorifera Vell.) e alecrim (Rosmarinus officinalis L.) sobre - gênero Candida. Rev Bras Plantas Med. $2011 ; 13(2): 203-8$.

3 Wingeter MA, Guilhermetti E, Shinobu CS, Takaki I, Svidzinski TIE. Identificação microbiológica e sensibilidade in vitro de Candida isoladas da cavidade oral de indivíduos HIV positivos. Rev Soc Bras Med Trop. 2007 mai-jun;40(3):272-6.

4 Giolo MP, Svidzinski TIE. Fisiopatogenia, epidemiologia e diagnóstico laboratorial da candidemia. J Bras Patol Med Lab. 2010 jun;46(3):225-34.

5 Tamura NK, Negri MFN, Bonassoli LA, Svidzinski TIE. Fatores de virulência de Candida spp isoladas de cateteres venosos e mãos de servidores hospitalares. Rev Soc Bras Med Trop. 2007 jan-fev; 40(1):91-3.

6 Samaranayake YH, Dassanayake RS, Jayatilake JAMS, Cheung BPK, Yau JYY, Yeung KWS, et al. Phospholipase B enzyme expression is not associated with other virulence attributes in Candida albicans isolates from patients with human immunodeficiency virus infection. J Med Microbiol. 2005 Jun;54(Pt 6):583-93.

7 Sahu JK, Ganguly S, Kaushik A. Triazoles: a valuable insight into recent developments and biological activities. Chin J Nat Med. 2013 Sep;1 1 (5):456-65.

8 Srinivasan A, Lopez-Ribot JL, Ramasubramanian AK. Overcoming antifungal resistance. Drug Discov Today Technol. 2014 Mar; 1 1:65-71.

9 Drago L, De Vecchi E, Nicola L, Gismondo MR. In vitro evaluation of antibiotics' combinations for empirical therapy of suspected methicillin resistant Staphylococcus aureus severe respiratory infections. BMC Infect Dis. 2007 Sep;7:111.
10 Mitsugui CS, Tognim MCB, Carrara-Marrone $\mathrm{FE}$, Garcia LB. Efeito antimicrobiano in vitro da associação de polimixina B e ceftazidima em amostras clínicas de Pseudomonas aeruginosa. Cienc Cuid Saude. 2008;7(supl 1):76-81.

11 Fuentefria AM, Andrade SF, Silveira GP, Kulkamp I, Pippi B, Machado MM, et al. Caracterização do perfil de susceptibilidade a antifúngicos azólicos de uma micoteca como embasamento para estratégias de combate a candidemias. J Infect Control. $2016 ; 5(1): 1-13$.

12 Lima IO, Oliveira RAG, Lima EO, Farias NMP, Souza EL. Atividade antifúngica de óleos essenciais sobre espécies de Candida. Rev Bras Farmacogn. 2006 abr-jun;16(2):197-201.

13 Baker P, Hill PJ, Snarr BD, Alnabelseya N, Pestrak MJ, Lee MJ, et al. Exopolysaccharide biosynthetic glycoside hydrolases can be utilized to disrupt and prevent Pseudomonas aeruginosa biofilms. Sci Adv. 2016 May;2(5):e1501632.

14 Chen Y, Ni W, Yan H, Qin XJ, Khan A, Liu H, et al. Spirostanol glycosides with hemostatic and antimicrobial activities from Trillium kamtschaticum. Phyłochemistry. 2016 Nov;131:165-73.

15 Eissa AM, Abdulkarim A, Sharples GJ, Cameron NR. Glycosylated nanoparticles as efficient antimicrobial delivery agents. Biomacromolecules. 2016;17(8):2672-9.

16 Armarego WLF, Perrin DD. Purification of laboratory chemicals. Oxford: Butterworth Heinemann; 1996.

17 Toshima K, Ishizuka T, Matsuo G, Nakata M. Practical glycosidation method of glycols using montmorillonite $\mathrm{K}-10$ as an environmentally acceptable and inexpensive industrial catalyst. Synlett. 1995; 1995(4):306-8.

18 Castro RD, Lima EO. Atividade antifúngica in vitro do óleo essencial de Eucalyptus globulus L. sobre Candida spp. Rev Odontol UNESP. 2010 mai-jun;39(3): 179-84.

19 Cortez LER, Yamaguchi MU, Cortez DAG, Pesco DCS. Avaliação da atividade antifúngica dos óleos essenciais de Lippia alba (Mill.) N. E. 
Brown (Verbenaceae) e Cymbopogon citratus (D.C.) Stapf (Poaceae). Mundo Saude. 2015 out-dez;39(4):433-40.

20 Clinical and Laboratory Standards Institute. M27-A3: reference method for broth dilution antifungal susceptibility testing of yeasts; approved standard. 3rd ed. Wayne: Clinical and Laboratory Standards Institute; 2008.

21 Lewis RE, Diekema DJ, Messer SA, Pfaller MA, Klepser ME. Comparasion of Etest, chequerboard dilution and time-kill studies for the detection of synergy or antagonism between antifungal agents tested against Candida species. I Antimicrob Chemother. 2002 Feb;49(2):345-51.

22 Hemaiswarya S, Kruthiventi AK, Doble M. Synergism between natural products and antibiotics against infectious diseases. Phytomedicine. 2008 Aug;15(8):639-52.

23 Silva F, Ferreira S, Duarte A, Mendonça DI, Domingues FC. Antifungal activity of Coriandrum sativum essential oil, its mode of action against Candida species and potential synergism with amphotericin B. Phytomedicine. 2011 Dec;19(1):42-7.

24 Pavia DL, Lampman GM, Kriz GS, Vyvyan JR. Introdução à espectroscopia. São Paulo: Cengage Learning; 2010.

25 De K, Legros J, Crousse B, Bonnet-Delpon D. Synthesis of 2,3-unsaturated glycosides via metal-free Ferrier reaction. Tetrahedron. 2008 Nov;64(46): 10497-500.

26 Macedo L, Fernandes T, Silveira L, Mesquita A, Franchitti AA, Ximenes EA. $\beta$-Lapachone activity in synergy with conventional antimicrobials against methicillin resistant Staphylococcus aureus strains. Phytomedicine. 2013 Dec;21(1):25-9.

27 Menezes TGC. Avaliação in vitro da atividade anti-MRSA de vancomicina encapsulada em lipossomas e do sinergismo com ácido úsnico e $\beta$-lapachona [dissertação]. Recife (PE): Universidade Federal de Pernambuco; 2014. $68 \mathrm{p}$.
28 Nogueira LG. Avaliação do potencial antimicrobiano de Pouteria spp. e de triterpenos quinonametídeos com enfoque no Helicobacter pylori [tese]. Araraquara (SP): Universidade Estadual Paulista Júlio de Mesquita Filho; 2012. 107 p.

29 Segatore B, Bellio P, Setacci D, Brisdelli F, Piovano $M$, Garbarino JA, et al. In vitro interaction of usnic acid in combination with antimicrobial agents against methicillinresistant Staphylococcus aureus clinical isolates determined by $\mathrm{FICl}$ and $\triangle \mathrm{E}$ model methods. Phytomedicine. 2012 Feb;19(3-4):341-7.

30 Barbosa DBM. Estudo da atividade antifúngica da associação do óleo essencial de Cymbopogon winterianus Jowitt (citronela) com antifúngicos sintéticos sobre espécies de Aspergillus [tese]. João Pessoa (PB): Universidade Federal da Paraíba; 2011.93 p.

31 Liu S, Hou Y, Chen X, Gao Y, Li H, Sun S. Combination of fluconazole with non-antifungal agents: a promising approach to cope with resistant Candida albicans infections and insight into new antifungal agent discovery. Int J Antimicrob Agents. 2014 May;43(5):395-402.

32 Cabral ME, Figueroa LIC, Fariña Jl. Synergistic antifungal activity of statin-azole associations as witnessed by Saccharomyces cerevisiae and Candida utilis-bioassays and ergosterol quantification. Rev Iberoam Micol. 2013 Jan;30(1):31-8.

33 Wilson DT, Drew RH, Perfect JR. Antifungal therapy for invasive fungal diseases in allogeneic stem cell transplant recipients: an update. Mycopathologia. 2009 Dec;168(6):313-27.

34 Rang HP, Dale MM, Ritter JM. Moore PK. Farmacologia. 6. ed. Rio de Janeiro: Elsevier; 2007.

35 Johnson MD, MacDougall C, Ostrosky-Zeichner L, Perfect JR, Rex JH. Combination antifungal therapy. Antimicrob Agents Chemother. 2004 Mar;48(3):693-715. 